Robert Primhak ${ }^{1}$, Nathalie Tabin², Nicole Beydon ${ }^{3}$, Jayesh Bhatt ${ }^{4}$, Ernst Eber ${ }^{5}$, Jürg Hammer ${ }^{6}$, Antonio Martinez-Gimeno ${ }^{7}$, Fabio Midulla ${ }^{8}$, Raffaella Nenna ${ }^{8}$, James Paton ${ }^{9}$, Robert Ross Russell10

\title{
Review
}

\section{Update of the European paediatric respiratory medicine syllabus}

The 10-year-old European syllabus for paediatric respiratory medicine (PRM; also known as paediatric pulmonology) was updated by a consensus-based method using an expert task force for redrafting, and a subsequent Delphi process to achieve consensus. There was a high degree of consensus for the final syllabus, which has been streamlined and made more relevant to current practice. All modules are now mandatory apart from the undertaking of research projects, which is optional. Although there are still a number of countries in Europe which do not recognise PRM as a separate subspecialty, there are paediatric respiratory physicians practising in every country in Europe, and a current and harmonised European syllabus in the subspecialty remains important for defining the training and areas of practice of PRM practitioners.

\section{Introduction}

Paediatric respiratory medicine (PRM; also known as paediatric pulmonology) has been recognised as a subspecialty in the USA since 1985 and by the Union of European Medical Specialists (UEMS) since 2015. The first European syllabus for PRM was agreed by a task force in 2002, and a more formal consensus-based syllabus was published in 2009 as part of the Harmonised Education and Training in Respiratory Medicine for European Specialists (HERMES) project. While this syllabus has been recognised by the UEMS, there are still a number of countries in Europe who do not recognise PRM as a separate subspecialty. Despite these bureaucratic variations, there are paediatric respiratory physicians practising in every country in Europe, and a harmonised European syllabus in the subspecialty remains important for defining the training and areas of practice of PRM practitioners.

\section{Project rationale}

After the PRM syllabus was published in 2009 [1, 2], a more detailed and prescriptive curriculum was published in 2010 [3]. In the modern world, all fields of medical practice are subject to rapid change and regular reviews of syllabi and curricula are a necessity if they are to remain relevant [4]. An update of the syllabus in PRM was thus necessary to maintain standards in training in the subspecialty and to ensure the

@ERSpublications

The European syllabus for paediatric respiratory medicine has been updated by a task force in conjunction with the ERS Paediatric Assembly and designated national experts. It has several new modules, and only one optional module remains. http://bit.ly/2KB9wCy

Cite as: Primhak R, Tabin N, Beydon N, et al. Update of the European paediatric respiratory medicine syllabus. Breathe 2019; 15: 173-180. 


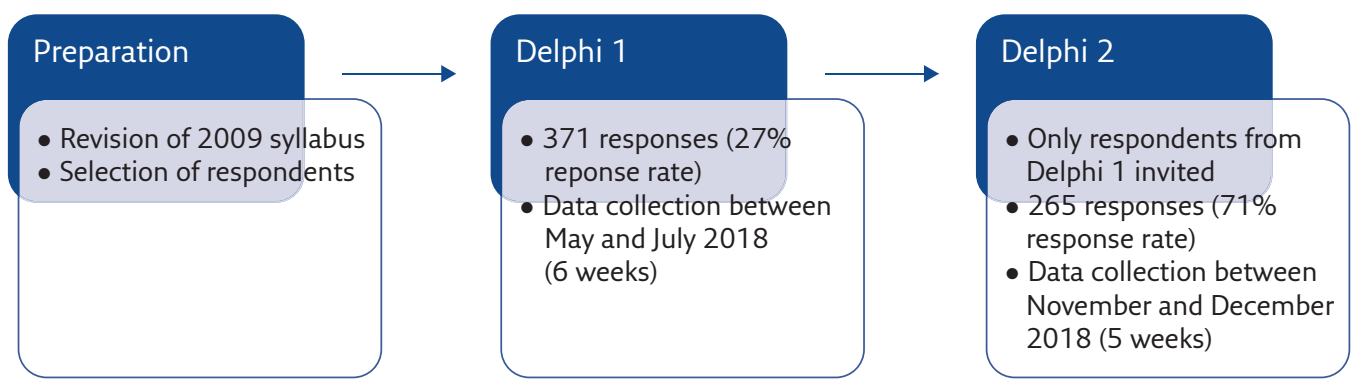

Figure 1 Delphi rounds.

continued relevance of the HERMES examination for paediatric specialists. Recognition of the training requirements by the UEMS also requires that they are regularly reviewed. A revision and update of the curriculum will also be necessary in due course. The adult respiratory medicine syllabus has also recently been updated and a similar procedure was adopted for this PRM syllabus update [5]

\section{Process and methods}

As the aim was to reach consensus amongst experts on the definition and structure of topics to be included in the syllabus, the Delphi technique, a group facilitation and communication process, was applied [6]. This technique, having been continuously enhanced through its use in previous European Respiratory Society (ERS) projects [7], includes quantitative and qualitative rounds of questions with interspersed face-toface meetings.

The first stage was a qualitative round that took place to draft the updated syllabus. The chair of the project, Robert Primhak, drafted a comparison between the existing syllabus, the ERS handbook of PRM [8] and a leading PRM reference book [9]. All task force members were then involved in the discussion of the content, which included topics which should be removed or introduced, and discussions on the structure of the syllabus. A final review took place with the chair and task force members to approve a first draft of the proposed new syllabus in the online tool "surveymonkey".

Two quantitative Delphi rounds were then carried out, as outlined in figure 1 . The respondents for the project were approached in two ways: 1) a group of "experts", comprising one or two representatives from member countries, nominated by their national paediatric pulmonary societies (where these exist); and 2) a general group comprising all members of ERS Assembly 7 (Paediatrics).
Respondents were asked if the modules should be either included or not included and, if included, whether they should be mandatory or optional. They were required to indicate their extent of agreement towards the inclusion of individual items on a Likert scale (from $1=$ strongly disagree to $5=$ strongly agree). An open box for comments was included at the end of each module. Consensus was then measured by the proportion of respondents with responses in the top two weights (agree and strongly agree). Universally agreed proportions do not exist for Delphi studies. The cut-off rate for consensus for this project was determined as $80 \%$. Any items or modules below this level were discussed.

\section{Results and discussion}

\section{Differences from the previous syllabus}

The initial task force revision included some structural changes from the original syllabus. A single separate module on "structure and function of the respiratory system" was introduced instead of repeating the topic in other modules throughout the syllabus; a separate module on "respiratory consequences of systemic/extrapulmonary conditions" was created to separate these often common problems, such as obesity, from those contained within the module on "rare diseases"; and the module on rehabilitation was renamed as "long-term management of chronic respiratory disorders" and transplantation issues were moved to appear within it. In addition, it was decided to define only those areas that were relevant to the trained specialist in PRM, and to dispense with the different levels used in the 2009 syllabus and thus the modules on management, teaching and communication were removed from the syllabus; knowledge of education theory was felt to be outside the scope of mandatory training in PRM, and much of the other content in these modules were considered as generic skills that were not specific to PRM. 


\section{Delphi responses}

Overall, the PRM Delphi 1 gathered 371 responses (27\% response rate). Only the 371 respondents from Delphi 1 were invited to participate in the Delphi 2, which gathered 265 respondents (71.43\% response rate). The certification status of respondents for the two rounds are shown in table 1. Approximately $45 \%$ of respondents in each round held an academic position, and 31 different European countries were represented.

In the first Delphi round respondents were asked "Do you think this module should be included as mandatory, optional or not included". There were 20 modules in the draft syllabus, and eight failed to achieve at least $80 \%$ agreement as mandatory modules, including "airway endoscopy", "allergic disorders" and "respiratory presentation or complications of systemic/extrapulmonary conditions". The combined question on the relevance of the module and its characteristic as mandatory or not, caused confusion and the task force decided to rephrase it. In the second Delphi round, the question was split in two, with the second part of the question asked at the end of the module for the sake of clarity:

- Do you think this module should be: included or not included?

- Do you think the module should be: mandatory or optional?

There were a total of 124 items within these modules, of which nine (7.3\%) failed to reach consensus. After discussion by task force members, three of these items ("atopic dermatitis", "food allergy", and "specific immunotherapy") were removed, and a broader topic "diagnosis and basic management of associated allergic conditions" was included.

In the second Delphi round all 20 modules reached at least $80 \%$ consensus for inclusion. Six modules did not reach consensus as mandatory modules. The agreement ratings for making these modules mandatory were as follows: "allergic disorders" $79.0 \%$; "sleep medicine and breathing control disorders" 73.8\%; "long-term management of chronic respiratory disorders" 74.7\%; "technology-dependent children" $77.6 \%$; "epidemiology and environmental health" 70.4\%; "evidence-based medicine and research" 62.5\%. The task force felt that this might reflect difference in clinical practice across different European countries, but could not defend the removal of any of these modules from the "mandatory" requirements. However, there was discussion about the components of the module on "evidencebased medicine and research". It was felt to be imperative that any specialist was capable of evaluating the scientific literature and had a basic understanding of the ethical principles involved in
Table 1 Certification status of respondents in the two Delphi rounds

\begin{tabular}{lcc}
\hline Certification status & Delphi 1 & Delphi 2\# \\
\hline Trainee & $38(10.1 \%)$ & $17(7.0 \%)$ \\
Certified $<5$ years & $71(19.1 \%)$ & $52(21.3 \%)$ \\
Certified $>5$ years & $234(63.1 \%)$ & $155(63.5 \%)$ \\
Other $(e . g$. no certification, & $28(7.5 \%)$ & $20(8.2 \%)$ \\
$\quad$ informal recognition) & 371 & 244 \\
Total & \\
\#: Not all Delphi 2 respondents answered this question.
\end{tabular}

research. However, there was less unanimity about the need for every specialist to have been an active researcher, so this item was split off into a separate optional module.

There were 122 items in the redrafted syllabus, of which five $(4.1 \%)$ failed to reach consensus in the second round, as detailed below.

\section{Discussion}

"Questionnaires in clinical assessment" was a debated topic, as some of the respondents did not use these or were unsure as to what these referred to. The task force decided to retain the item in the syllabus as it was felt that a basic knowledge of such questionnaires, including strengths and limitations was essential to contemporary practice.

"Equipment maintenance, hygiene and infection control during test procedures" only reached $79.3 \%$ consensus, but again was felt to be an essential patient safety item which could not be discarded or made optional.

"Induced sputum testing" was felt to be common enough in modern clinical practice to be included, noting the $79.7 \%$ agreement.

"Assessing respiratory risk of air travel, altitude and diving" also raised some discussion, due to a consensus rate below $70 \%$ after the first round. The item was then rephrased, from the initial name of "fitness to fly" to the current topic name with the content broadened to include other risks. Even though the consensus rate of $77 \%$ did not reach the cut-off rate of $80 \%$ during the second round, the group decided to keep the item as these assessments are frequently requested tests in respiratory laboratories in many European countries.

"Psychological evaluation tools (including quality of life)" reached $76.6 \%$ consensus, but again the panel felt that a basic understanding of quality of life tools was important in understanding the scientific literature.

The final draft syllabus contained 21 modules and 122 items, outlined in table 2. 
Table 2 Paediatric respiratory medicine syllabus 2019

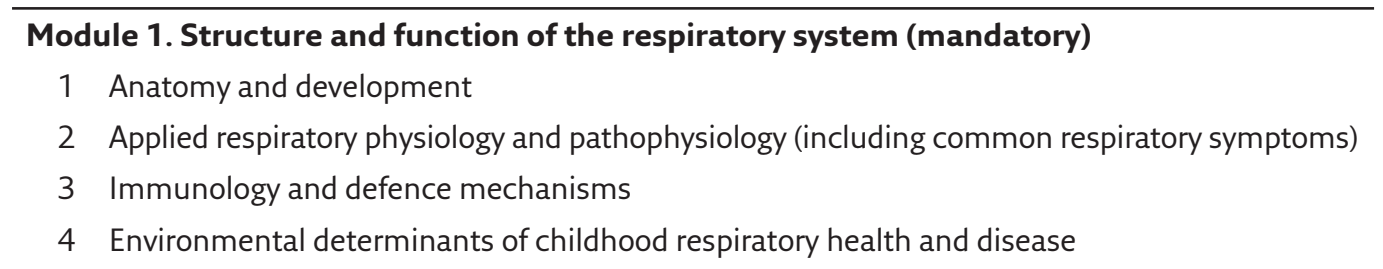

Module 2. Evaluation of respiratory symptoms and signs (mandatory)

Evaluation and management of:

1 Acute and chronic cough

2 Stridor, wheezing and other respiratory noises

3 Dyspnoea and breathlessness

4 Respiratory distress and respiratory insufficiency

5 Questionnaires in clinical assessment

Module 3. Pulmonary function and diagnostic testing (mandatory)

Measurement, performance and interpretation of:

1 Volume-time and flow-volume curves

2 Static lung volumes

3 Respiratory mechanics

4 Reversibility testing

5 Bronchial provocation testing

6 Cardiopulmonary exercise testing

7 Blood gases and oximetry

8 Lung diffusion

9 Ventilation homogeneity

10 Respiratory muscle function

11 Equipment maintenance, hygiene and infection control during test procedures

12 Definitions of measured indices

13 Choice and appropriate use of reference values

14 Test variability and reproducibility

15 Quality control in paediatric lung function laboratories

16 Health and safety issues in relation to paediatric lung function testing

17 Lung function in non-cooperative children

Additional diagnostic tests

Measurement, performance and interpretation of:

1 Exhaled nitric oxide measurements

2 Induced sputum testing

3 Assessing respiratory risk of air travel, altitude and diving

\section{Module 4. Airway endoscopy (mandatory)}

1 Organisation of an endoscopy suite including equipment maintenance and hygiene

2 Indications for, contraindications, performance and interpretation of flexible bronchoscopy and associated procedures

3 Sedation and anaesthesia for paediatric flexible bronchoscopy

4 Indications for and contraindications of interventional bronchoscopy techniques other than foreign body removal 
Table 2 Continued

5 Indications for and contraindications of rigid bronchoscopy

6 Evaluation and management of risks and complications of airway endoscopy

\section{Module 5. Imaging (mandatory)}

1 Indications for, interpretation and basic principles of conventional radiography, computed tomography, magnetic resonance imaging, ultrasonography and isotope imaging methods

2 Comparative radiation burden of the different procedures

3 Indications for interventional radiology

\section{Module 6. Respiratory infections (mandatory)}

Epidemiology, diagnosis, management and complications of:

1 Acute upper respiratory tract infections

2 Acute lower respiratory tract infections

3 Pleural infections

4 Tuberculosis (TB) and non-TB mycobacterial diseases

5 Diagnosis and management of respiratory infections in high-risk situations

6 Diagnosis and management of protracted bacterial bronchitis and non-CF bronchiectasis

7 Lung involvement in the immunocompromised host

8 Microbiology and infectivity

9 Pharmacology of antimicrobial and antiviral drugs

10 Immunisations for respiratory pathogens

11 Accuracy and interpretation of microbiological tests

\section{Module 7. Asthma and wheezing disorders (mandatory)}

1 Different phenotypes and their different pathologies and long-term outcomes (including underlying pathophysiology and basic epidemiology)

2 Environmental factors relevant to asthma and other wheezing disorders

3 Difficulties in diagnosis and differential diagnosis

4 Management of asthma at different ages including age-related pharmacology, and pharmacological and non-pharmacological management

5 Management of difficult and severe asthma

\section{Module 8. Allergic disorders (mandatory)}

Epidemiology, diagnosis and management of:

1 Anaphylaxis

2 Allergic rhinitis

3 Bronchopulmonary aspergillosis

4 In vivo and in vitro testing for allergic disorders

5 Prevention measures

6 Diagnosis and basic management of associated allergic conditions

\section{Module 9. Cystic fibrosis (CF) (mandatory)}

1 Epidemiology, genetics, pathophysiology and prognosis

2 Screening and diagnosis

Epidemiology, diagnosis, management and complications of:

3 CF lung disease

4 Extrapulmonary manifestations of $\mathrm{CF}$ including nutrition

5 Microbiology relevant to $C F$, including cross-infection 
Table 2 Continued

\section{Module 10. Congenital malformations (mandatory)}

1 Epidemiology, diagnosis and management of congenital malformations affecting the respiratory system

2 Antenatal diagnosis and management

3 Follow-up and long-term outcomes

\section{Module 11. Bronchopulmonary dysplasia/chronic neonatal lung disease (mandatory)}

1 Epidemiology, aetiology, prevention, diagnosis, management and complications

2 Follow-up and long-term outcomes

\section{Module 12. ENT (ear, nose and throat) and aerodigestive-related respiratory problems (mandatory)}

1 Aspiration due to disorders of feeding and swallowing

2 Gastro-oesophageal reflux

3 Laryngeal and tracheal disorders

4 Foreign body inhalation

\section{Module 13. Respiratory presentation or complications of systemic/extrapulmonary conditions (mandatory)}

1 Primary immunodeficiency syndromes

2 Secondary immunodeficiency syndromes/immunosuppression

3 Cardiac disease

4 Gastrointestinal, liver and kidney disease

5 Haematological disease

6 Obesity

7 Connective tissue diseases

8 Musculo-skeletal and neuromuscular diseases

\section{Module 14. Rare diseases (mandatory)}

Pathophysiology, genetics, aetiology, diagnosis and management of:

1 Primary ciliary dyskinesia

2 Bronchiolitis obliterans

3 Interstitial lung diseases

4 Pulmonary vascular disorders including pulmonary arterial hypertension

5 Pulmonary haemorrhage

6 Pleural diseases including spontaneous pneumothorax

7 Drug- and radiation-induced lung diseases

8 Other rare lung diseases

\section{Module 15. Respiratory sleep medicine and breathing control disorders (mandatory)}

1 Physiology and pathophysiology of sleep relevant for paediatric respiratory medicine

2 Indications and interpretation of the results of polysomnography

Epidemiology, aetiology, diagnosis of, screening for, and complications of:

3 Obstructive sleep apnoea syndromes

4 Central sleep apnoea/hypoventilation

5 Dysfunctional breathing/hyperventilation syndromes 
Table 2 Continued

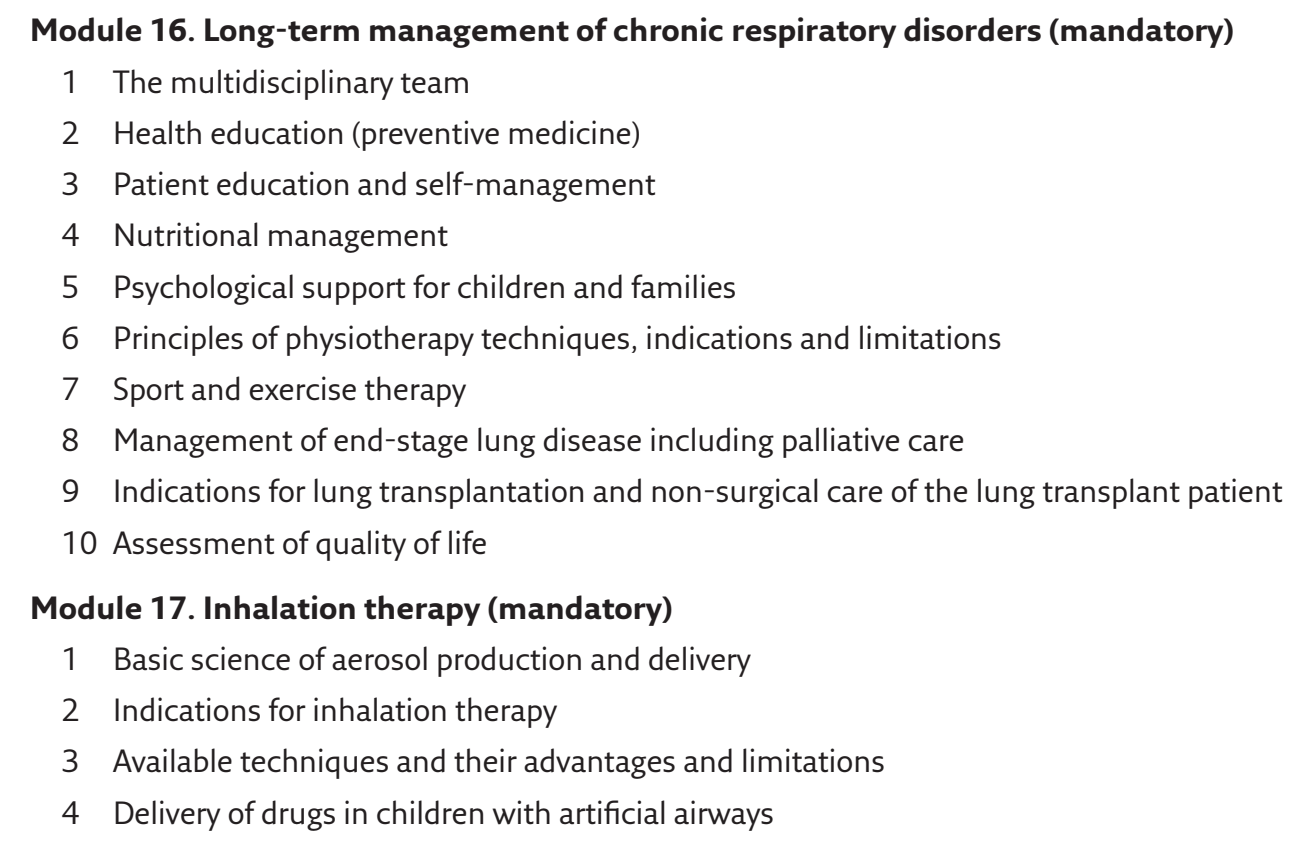

\section{Module 18. Technology-dependent children (mandatory)}

Management, monitoring and weaning of:

1 Home oxygen therapy

2 Invasive and noninvasive home ventilatory support

3 Tracheostomy

4 Basic technical understanding of equipment

5 Airway clearance techniques

6 Recognition of associated problems (including neurodevelopmental assessment)

\section{Module 19. Epidemiology and environmental health (mandatory)}

1 Basic understanding of epidemiological principles

2 Impact of indoor and outdoor air pollution on respiratory health

3 Smoking impact, prevention and cessation

4 The burden of paediatric respiratory diseases on healthcare resources

\section{Module 20. Evidence-based medicine (mandatory)}

1 Scientific literature appraisal

2 Understanding and application of the ethical principles of paediatric research

\section{Module 21. Research (optional)}

1 Basic understanding and application of the principles of planning, designing, conducting, analysing and publishing research projects

\section{Conclusion}

Both Delphi rounds achieved a reasonable response rate from a broad group of respondents, and a relatively high degree of consensus on the content of the updated syllabus; most of the modules were agreed to be mandatory for training in PRM. In our view, through the process of revision and review, the new syllabus has been streamlined and made more relevant to current practice. This process will need to be repeated within the next 10 years to ensure that the syllabus and training programmes in Europe continue to be relevant to the needs of tomorrow's patients. 


\section{Affiliations}

Robert Primhak ${ }^{1}$, Nathalie Tabin ${ }^{2}$, Nicole Beydon ${ }^{3}$, Jayesh Bhatt ${ }^{4}$, Ernst Eber 5 , Jürg Hammer 6 , Antonio Martinez-Gimeno7, Fabio Midulla ${ }^{8}$, Raffaella Nenna ${ }^{8}$, James Paton ${ }^{9}$, Robert Ross Russell ${ }^{10}$

${ }^{1}$ Assessments Director, European Respiratory Society. ${ }^{2}$ Education Dept, European Respiratory Society, Lausanne, Switzerland. ${ }^{3}$ Functional Unit of Pulmonary Function and Sleep Testing, Armand Trousseau Hospital, Paris, France. ${ }^{4}$ Nottingham Children's Hospital, Nottingham University Hospitals, Nottingham, UK. ${ }^{5}$ Division of Paediatric Pulmonology and Allergology, Dept of Paediatrics and Adolescent Medicine, Medical University of Graz, Graz, Austria. ${ }^{6}$ Division of Intensive Care and Pulmonology, University Children's Hospital Basel, Basel, Switzerland. ${ }^{7}$ Dept of Paediatrics, Complejo Hospitalario Universitario de Toledo and Universidad de Castilla La Mancha, Toledo, Spain. ${ }^{8}$ Dept of Paediatrics and Maternal Health, Sapienza University of Rome, Rome, Italy. ${ }^{9}$ School of Medicine, College of Medical, Veterinary, and Life Sciences, University of Glasgow, Glasgow, UK. ${ }^{10}$ Dept of Paediatrics, Cambridge University Hospital, Cambridge, UK.

\section{Conflict of interest}

R. Primhak has nothing to disclose. N. Tabin is an employee of the European Respiratory Society. N. Beydon has nothing to disclose. J. Bhatt has nothing to disclose. E. Eber has nothing to disclose. J. Hammer has nothing to disclose. A. Martinez-Gimeno has nothing to disclose. F. Midulla has nothing to disclose. R. Nenna has nothing to disclose. J. Paton has nothing to disclose. R. Ross Russell has nothing to disclose.

\section{References}

1. Zach MS, Long Range Planning Committee, Paediatric Assembly of the European Respiratory Society, Committee on Paediatric Respiratory Training in Europe, European Board of Paediatrics. Paediatric respiratory training in Europe: syllabus and centres. Eur Respir J 2002; 20: 1587-1593.

2. Gappa M, Noel J-L, Severin T, et al. Paediatric HERMES: a European Syllabus in Paediatric Respiratory Medicine. Breathe 2009; 5: 236-247.

3. Gappa M, Noel J-L, Severin T, et al. Paediatric HERMES European curriculum recommendations for training in paediatric Respiratory Medicine. Breathe 2010; 7: 72-79.

4. General Medical Council (UK). Excellence by Design. 2017. www.gmc-uk.org/education/postgraduate/standards_for_ curricula.asp
5. Tabin N, Mitchell S, O'Connell E, et al. Update of the ERS international Adult Respiratory Medicine syllabus for postgraduate training. Breathe 2018; 14: 19-28.

6. Thangaratinam S, Redman C. The Delphi technique. Obstet Gynaecol 2005; 7: 120-125.

7. Aliberti, S. ERS curriculm design: a summary of projects. www.ersnet.org/professional-development/ers-curriculumdesign-a-summary-of-projects Date last accessed: April 12, 2019.

8. Eber E, Midulla F, eds. Paediatric Respiratory Medicine. 1st Edn. Sheffield, European Respiratory Society, 2013.

9. Wilmott RW, Boat TF, Bush A, eds. et al. Kendig and Chernick's Disorders of the Respiratory Tract in Children. 8th Edn Philadelphia, Elsevier, 2012. 